\title{
The Application of Computer Aided Analysis in Graphic Design of Visual Aesthetics
}

\author{
Qiu Hong-Li ${ }^{1,}$, , Wang Hong-Lei ${ }^{1, b}$ \\ ${ }^{1}$ Henan Quality Engineering Vocational College, Pingdingshan HeNan 467001, China \\ a315057749@qq.com, b281912303@qq.com
}

\begin{abstract}
Keywords: graphic design; design geometry; computer aided design; aesthetics
\end{abstract}
\begin{abstract}
Order beauty is the core of graphic design, with its good works follow the rules of th11e performance of the order (such as symmetry, golden section, etc.) consciously or unconsciously. In graphic design, these rules reflect the basic principles for the design geometry whose proper use can make the design more harmonious, symmetry and balance in vision. Therefore, an important aspect to evaluate graphic design work is to look at its proportion and structure among each element. Aiming at the current problem that graphic design software is unavailable to provides user's design with support in the composition theory side. This paper designed a computer aided analysis system of Graphic design visual aesthetics based on design geometry, which realized such basic functions as the interactive segmentation and acquisition of the import of graphic design work and elements, and analysis and judgment of the relationship between proportion and composition. The system can be used in the design of the visual aesthetic evaluation of the work, can also be engaged in graphic design to provide the users with design support as relevant geometric aesthetic principles.
\end{abstract}

\section{Preface}

Graphic design works characterized by simple graphics language, strong artistry, easy to spread and is able to fully reflect the author's intention and artistic style. Even if there is a rapid development of multi-media and wide application of 3D technology today, graphic still has a strong vitality. From the point of view of its type, graphic design includes: painting, photography, posters, print ads and even calligraphy.

Order beauty is the core of graphic design, with its good works follow the rules of th11e performance of the order (such as symmetry, golden section, etc.) consciously or unconsciously. In graphic design, these rules reflect the basic principles for the design geometry whose proper use can make the design more harmonious, symmetry and balance in vision. Therefore, an important aspect to evaluate graphic design work is to look at its proportion and structure among each element.

Computer aided design software provides a convenient graphic design platform for users, but current mainstream graphics design platform cannot provide support and judgment on design geometric theory for users' works . In the processing of graphics and design, the users still rely on their own personal artistic quality to judge and improve the work. At the same time, a large number of users proficient in computer graphics software have no clue or end up with a chaotic of disorder work, because the users themselves know nothing about design geometry knowledge. Therefore, This paper designed a computer aided analysis system of Graphic design visual aesthetics based on design geometry, which realized such basic functions as the interactive segmentation and acquisition of the import of graphic design work and elements, and analysis and judgment of the relationship between proportion and composition. The system can be used in the design of the visual aesthetic evaluation of the work, can also be engaged in graphic design to provide the users with design support as relevant geometric aesthetic principles.

\section{Basic theory of geometric aesthetics}

\section{Design geometry}

In nature, there are a variety of natural laws, such as the proportion of the human body, and the 
growth of plants and animals in accordance with the 1:1.618 of the golden section ratio. These laws of natural proportions provide the basic starting point for the works of artists and designers. The use of the basic principles of these geometries can make the design of the work in the visual feel more harmonious, symmetrical and balanced.

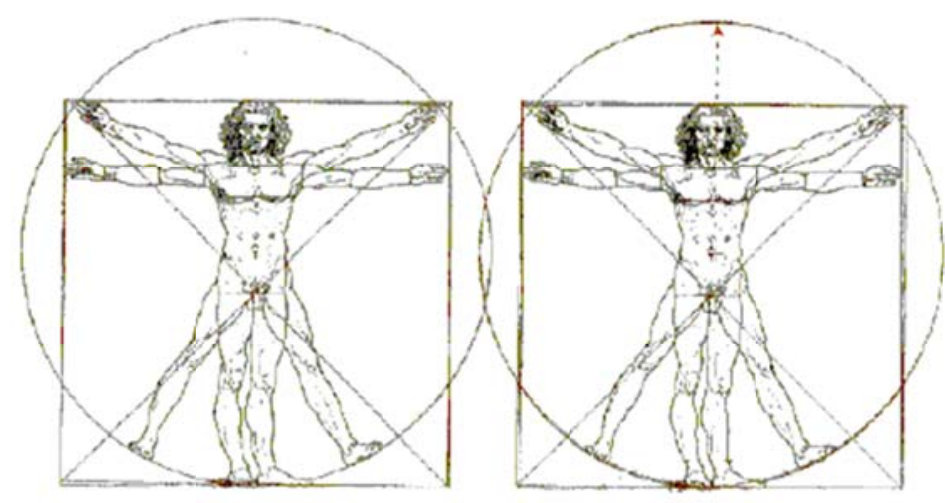

Figure 1 Human height is consistent with the navel as the segmentation point of the golden ratio

\section{Golden ratio}

Figure 2 painting works in strict accordance with the golden ratio

Divide a line segmen into two parts, make the ratio of the longer line segment $\mathrm{AC}$ to the whole line segment $\mathrm{AB}$ equal to the ratio of longer line segment $\mathrm{AC}$ to shorter line segment $\mathrm{BC}$. The proportion of this line segment is approximately 1.618:1, which is called golden ratio. Golden ratio has a perfect proportion.

In this ratio, different golden section of the rectangle, golden section of the triangle, golden section of the oval shape and the golden section of the spiral can be obtained.The shapes with similar aesthetic nature can be fused to greater overall pattern with its original features maintained and bring forth a more harmonious and natural overall picture.

\section{Dynamic rectangle}

In addition to the golden section, the dynamic rectangle such as the ones with irrational fraction can also produce a series of visual pleasure of the plane ratio. These rectangles and the golden section are called dynamic rectangles, and they can produce a lot of segmentation and combination, whose graphics are always in line with the proportion of the original rectangle.

\section{Computer aided analysis of graphic design visual aesthetics}

The above geometric structure principles be followed, a sense of cohesion is introduced into the graphic design works with the result that each element of the work has a visual sense of belong.

These principles are not only the key to understanding a large number of graphic design works, but also the aesthetic indicators of the analysis and evaluation of graphic design works. In this paper, 
a computer aided analysis system of graphic design based on the principle of geometric aesthetics is presented.

System framework

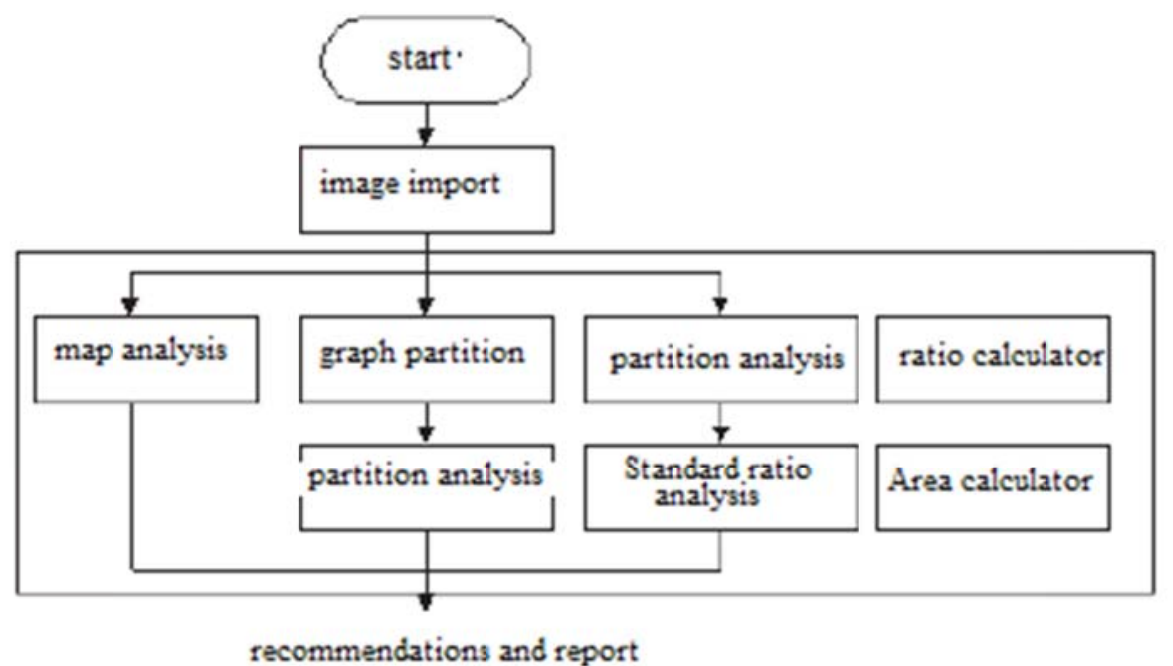

\section{Figure analysis}

Fig. 3 computer aided analysis system framework of graphic design visual aesthetics

After images is imported onto the system, firstly, the image is placed at the system layer and bottom locked, which can make the image can't be arbitrarily modified. To arrange the image on the bottom can guarantee the needed auxiliary line, template superimposed on top of the image without being blocked in the following analysis .

After the above treatment, mappable unit analysis can be conducted by obtaining the image attributes. First of all, to judge whether the image is horizontal or vertical, and then gets the picture insertion point and its length and width and calculate the contour ratio.

Contours ratio have been obtained, compare the value with such dynamic rectangle proportion as the square root of 2, golden ratio, square root of 3,4,5. Judge the degree of conformity within acceptable proportion precision. Regarded it as the standard value when the error values in a certain range, while if the value of messenger is not in this range, prompt contours scale and standard value. If the obtained contour proportion is near the standard values, calculated the length and width values in the standard value and provide suggestions for revision to indicate value increases or decreases in the value of the image and the standard difference and suggestions.

\section{Split line analysis}

Draw the split line by human-computer mode and calculate the proportional relation of split line and the whole image, split line to another through linear system elements (linear, rectangular, etc.) exist in the graphic design when there is obvious segmentation among the graphic elements .Split line analysis can be carried out by continuous split line distance ratio analysis and reference distance ratio analysis. The former is to obtain proportional relation between the adjacent two split line, and the latter is to obtain the relative proportions of each split line to a reference distance.

\section{Standard auxiliary line template}

If there is no split line in the image, or the analysis of the proportion of the split line must be carried out after the end of the analysis, the image can be analyzed further by the standard auxiliary line analysis template. Due to the complexity of the dynamic rectangle segmentation, it is difficult to find the use of dynamic rectangle in artificial judgment, and the standard auxiliary line template can be used to explore the deep level of the relationship.

System provided dynamic rectangle including a golden square root of 2, 3,4,5 and more than 80standard auxiliary line template. Reasonable selection can be made according to map contour and distribution of image elements. 


\section{Auxiliary tools}

The system provides a scale calculator and an area calculator, which can be used at any time during the analysis process, and the area calculator can easily calculate the area of the regular and irregular figure in the image.

\section{Report}

The system provides report function, output and save all the analysis results and revision suggestion. Avoiding repeated work in the future, the users can carry out the investigation and research much more easily.

\section{System implementation}

According to the framework of the system, this paper realized a prototype system on the platform of AutoCAD. With the help of the AutoLIST language, this paper realize direct access and modification to AutoCAD Graphics, and real-time modification and addition of screen graphics, and the system menu and dialog box are redesigned. The system can complete the introduction of graphic design works, the interactive segmentation and acquisition of design elements, the analysis and judgment of the relationship between the proportion and the composition.

\section{Summary}

This paper designed a computer aided analysis system of Graphic design visual aesthetics based on design geometry, which realized such basic functions as the interactive segmentation and acquisition of the import of graphic design work and elements, and analysis and judgment of the relationship between proportion and composition. The system can be used in the design of the visual aesthetic evaluation of the work, can also be engaged in graphic design to provide the users with design support as relevant geometric aesthetic principles.

Prototype system makes full use of the system function provided by AutoCAD. However, AutoCAD, after all, is only an engineering drawing software instead of a special graphic design software. Therefore, how to transplant it to professional graphic design system is the focal point of work of the next step.

\section{References}

[1] Li Jinwang. Research on the combination and application of the design software in computer graphic design [J]. Shandong industrial technology. 2015 (09)

[2] Li Tao, Zhang Zhigang. An analysis of the art pursuit of computer graphic design [J]. Journal of Handan College. 2006 (03)

[3] Bian Ying. The impact of software on the graphic design [J]. public art. 2014 (20)

[4] He Xie Fei. Research and discussion of [J]. computer knowledge and technology of graphic design teaching. 2014 (35) 